\title{
Deep GMRT radio observations and a multi-wavelength study of the region around HESS J1858+020
}

\author{
J. M. Paredes ${ }^{1}$, C. H. Ishwara-Chandra ${ }^{2}$, V. Bosch-Ramon ${ }^{1}$, V. Zabalza ${ }^{3}$, K. Iwasawa ${ }^{4}$, and M. Ribó ${ }^{1}$ \\ 1 Departament d'Astronomia i Meteorologia, Institut de Ciències del Cosmos, Universitat de Barcelona, IEEC-UB, \\ Martí i Franquès 1, 08028 Barcelona, Spain \\ e-mail: jmparedes@ub.edu; [vbosch;mribo]@am.ub.es \\ 2 National Centre for Radio Astrophysics, TIFR, Post Bag 3, Ganeshkhind, 411007 Pune, India \\ e-mail: ishwar@ncra.tifr.res.in \\ 3 Max-Planck Institut für Kernphysik, Postfach 103980, 69029 Heidelberg, Germany \\ e-mail: Victor.Zabalza@mpi-hd.mpg.de \\ ${ }^{4}$ ICREA, Institut de Ciències del Cosmos, Universitat de Barcelona, IEEC-UB, Martí i Franquès 1, 08028 Barcelona, Spain \\ e-mail: kazushi .iwasawa@icc.ub.edu
}

Received 17 July 2013 / Accepted 10 November 2013

\begin{abstract}
Context. There are a number of very high energy sources in the Galaxy that remain unidentified. Multi-wavelength and variability studies, and catalogue searches, are powerful tools to identify the physical counterpart, given the uncertainty in the source location and extension.

Aims. This work carries out a thorough multi-wavelength study of the unidentified, very high energy source HESS J1858+020 and its environs.

Methods. We have performed Giant Metrewave Radio Telescope observations at $610 \mathrm{MHz}$ and $1.4 \mathrm{GHz}$ to obtain a deep, low-frequency radio image of the region surrounding HESS J1858+020. We analysed archival radio, infrared, and X-ray data as well. This observational information, combined with molecular data, catalogue sources, and a nearby Fermi gamma-ray detection of unidentified origin, are combined to explore possible counterparts to the very high energy source.

Results. We provide with a deep radio image of a supernova remnant that might be related to the $\mathrm{GeV}$ and $\mathrm{TeV}$ emission in the region. We confirm the presence of an $\mathrm{H}$ II region next to the supernova remnant and coincident with molecular emission. A potential region of star formation is also identified. We identify several radio and X-ray sources in the surroundings. Some of these sources are known planetary nebulae, whereas others may be non-thermal extended emitters and embedded young stellar objects. Three old, background Galactic pulsars also neighbour HESS J1858+020 along the line of sight.

Conclusions. The region surrounding HESS J1858+020 is rich in molecular structures and non-thermal objects that may potentially be linked to this unidentified very high energy source. In particular, a supernova remnant interacting with nearby molecular clouds may be a good candidate, but a star forming region, or a non-thermal radio source of yet unclear nature, may also be behind the gamma-ray source. The neighbouring pulsars, despite being old and distant, cannot be discarded as candidates. Further observational studies are needed, however, to narrow the search for a counterpart to the HESS source.
\end{abstract}

Key words. techniques: interferometric - radio continuum: general - gamma rays: general - ISM: supernova remnants

\section{Introduction}

The High Energy Stereoscopic System (HESS) survey of the inner Galaxy (Aharonian et al. 2006) has revealed new sources that remain unidentified because they have not been associated with a particular object from which very high energy (VHE: $E>100 \mathrm{GeV}$ ) emission is expected. A study of the environment of eight of these unidentified extended $\mathrm{TeV}$ sources with high detection significance revealed no plausible counterpart (Aharonian et al. 2008). These eight sources are extended, with angular sizes ranging from 3 to 18 arcmin. Their spectra in the TeV energy range follow a power-law with a differential spectral index in the range 2.1 to 2.5 . The source sizes and spectra are similar to previously identified Galactic VHE sources (e.g., PWNe). In some of these sources, a plausible counterpart has been found (HESS J1731-347: Tian et al. 2010; HESS J1841-055: Pletsch et al. 2012) or has been described as ancient PWNe (e.g., HESS J1427-608; Tibolla et al. 2012) in the last few years, but other sources, such as HESS J1858+020, still lack a plausible counterpart at low energies.

The source HESS J1858+020 is one of the weakest gammaray sources of the Aharonian et al. (2008) sample and it shows a slight extension along its major axis, in the northsouth direction. Close to it lies HESS J1857+026, which is a larger and brighter source, although both sources are considered distinct objects because no significant emission connects them. The spectrum of HESS J1858+020, over the range $0.5-80 \mathrm{TeV}$, follows a power law of spectral index $2.17 \pm$ 0.12 and its flux is $3.5 \times 10^{-12} \mathrm{erg} \mathrm{cm}^{-2} \mathrm{~s}^{-1}$ (Aharonian et al. 2008). MAGIC $(E>100 \mathrm{GeV})$ observed HESS J1857+026 in 2010. In these observations, HESS J1858+020 was also detected although with low significance given the relatively low MAGIC exposure at $0.5^{\circ}$ angular distance to the pointing positions (Stamatescu et al. 2012). At GeV energies, 2FGL J1857.6+0211 is positionally the closest Fermi-LAT source to HESS J1858+020 that appears in the Fermi Large Area 
Telescope Second Source Catalogue (Nolan et al. 2012). It is placed at $10^{\prime}$ from the HESS J1858+020 location, with an error box radius for the source position of about $4^{\prime}$, an energy flux of $\approx 10^{-10} \mathrm{erg} \mathrm{cm}^{-2} \mathrm{~s}^{-1}$ from 0.1 to $100 \mathrm{GeV}$, and a power-law index of 2.16.

At lower energies, this region was explored in the VLA Galactic Plane Survey (VGPS) at $1.4 \mathrm{GHz}$ (Stil et al. 2006) with $1^{\prime}$ resolution. Using these data, Green (2009) identified the source G35.6-0.4 as a supernova remnant (SNR), which is placed in the north-west border of HESS J1858+020.

More recently, Paron et al. (2011) used the ${ }^{13} \mathrm{CO}(J=1-0)$ line from the Galactic Ring Survey (Jackson et al. 2006) to study the existence of molecular clouds towards the region of the SNR G35.6-0.4. They found a molecular cloud composed of two clumps, extending near the HESS source central region. The study of the southeastern clump revealed the existence of a young stellar object, but the lack of evidence of molecular outflows, which would represent a jet able to produce gamma rays (Araudo et al. 2007), seemed to discard the possibility of such an object as responsible of the observed $\mathrm{TeV}$ gamma-ray emission. It has also been proposed that this emission is produced by hadronic interactions between the molecular cloud atoms and protons accelerated by the shock front of the SNR G35.6-0.4 (Paron et al. 2011; Torres et al. 2003).

To identify the (possible) radio counterpart of HESS J1858+020, we conducted exploratory observations with the Giant Metrewave Radio Telescope (GMRT) at $1.4 \mathrm{GHz}$ and $610 \mathrm{MHz}$ frequencies. These observations provide us with deep radio maps of the field of HESS J1858+020 with arcsecond detail and improve the rms of the currently available images of the NVSS (Condon et al. 1999) by one order of magnitude. As the data were taken at two different epochs, we can search for radio variability that is expected to be found in compact gamma-ray emitters such as microquasars and blazars.

In this work, we interpret the radio data from the region of the high energy source HESS J1858+020 in a multi-wavelength context, in which the available IR, X-ray, and gamma-ray data are also included. The paper is structured as follows. In Sects. 2 and 3 , we describe the radio observations, the data reduction, and the multi-wavelength archival data used; in Sects. 4 and 5, we present our results, and we discuss them in Sect. 6 .

\section{The GMRT observations}

We observed the HESS J1858+020 region at $610 \mathrm{MHz}(49 \mathrm{~cm})$ and $1.4 \mathrm{GHz}(21 \mathrm{~cm})$ using the GMRT, located in Pune (India). The radio observatory GMRT is an array of 30 antennas of $45 \mathrm{~m}$ in diameter each spread over distances of up to $25 \mathrm{~km}$. We carried out the observations in three different epochs: May, June and July 2009 (see Table 1). The angular size of our target $\mathrm{TeV}$ source is about $10^{\prime}$, which is well within the primary beam (FWHM) of GMRT at both $1.4 \mathrm{GHz}\left(24^{\prime}\right)$ and $610 \mathrm{MHz}\left(43^{\prime}\right)$. The total observing time devoted to each frequency is detailed in Table 1 . Observations were made with two 16-MHz sidebands (USB and LSB) centred at $610 \mathrm{MHz}$ and 1.4 GHz. The USB and LSB data were edited separately in AIPS $^{1}$. The LSB data were of relatively poor quality at $610 \mathrm{MHz}$, hence not included in the final images. The flux density scale was set using 3C 286 and 3C 48 as primary amplitude calibrators, and the phase calibration was performed by

\footnotetext{
1 The NRAO Astronomical Image Processing System. http://www . aips.nrao.edu/
}

Table 1. Log of the GMRT observations of HESS J1858+020.

\begin{tabular}{lccccc}
\hline \hline $\begin{array}{l}\text { Date } \\
2009\end{array}$ & $\begin{array}{c}\text { Frequency } \\
{[\mathrm{MHz}]}\end{array}$ & $\begin{array}{c}\text { Beam size } \\
{\left[{ }^{\prime \prime}\right] \times\left[^{\prime \prime}\right]}\end{array}$ & $\begin{array}{c}\text { PA } \\
{\left[{ }^{\circ}\right]}\end{array}$ & $\begin{array}{c}\text { rms noise } \\
\text { mJy beam }^{-1}\end{array}$ & $\begin{array}{c}\text { ON } \\
\text { source } \\
{[\mathrm{h}]}\end{array}$ \\
\hline May 31 & 1400 & $3.3 \times 3.0$ & 27 & 0.049 & 5.0 \\
June 4 & 610 & $16.8 \times 5.1$ & 43 & 0.26 & 5.0 \\
July 8 & 610 & $10.9 \times 4.5$ & 56 & 0.24 & 4.5 \\
June 4 + July 8 & 610 & $12.2 \times 4.8$ & 52 & 0.22 & 9.5 \\
\hline
\end{tabular}

observing repeatedly the phase calibrator $1822-096$ at both frequencies. A few iterations of phase-only self-calibration and one iteration of amplitude and phase self-calibration were performed to improve the image quality. We have produced different maps for each frequency and run (day).

To detect variability, we observed at two different epochs at $610 \mathrm{MHz}$, which allowed us to identify any possible variable radio source within the HESS J1858+020 region observed. By averaging the two runs at $610 \mathrm{MHz}$, we also produced a deeper radio image at this frequency.

\section{Multi-wavelength archival data}

We explored the IR band by making use of data products at 3.4, 4.6, 12, and $22 \mu \mathrm{m}$ from the Wide-field Infrared Survey Explorer (WISE; Wright et al. 2010). We explored the X-ray band by analysing archival Chandra data covering the central region of HESS J1858+020. The X-ray data correspond to the project ObsID9116, and consist of $29.8 \mathrm{ks}$ exposure observations carried out with the detector ACIS-I in mode VFAINT on June 21, 2008. The high energy gamma-ray data were obtained from the Fermi-LAT Second Source Catalogue (2FGL; Nolan et al. 2012). The information about the molecular cloud distribution in the region was obtained from the Galactic Ring Survey (Jackson et al. 2006).

\section{Radio sources around HESS J1858+020}

\subsection{GMRT results}

Figure 1 shows the two epochs average $610 \mathrm{MHz}$ GMRT radio image (black contours) of the field of HESS J1858+020 with several arc second resolution. This $610 \mathrm{MHz}$ GMRT image is the most sensitive (few mJy beam ${ }^{-1}$ ) and the highest resolution image ever obtained of this region. The colour image shows the VGPS data of this region at $1.4 \mathrm{GHz}$ with arc minute resolution (Stil et al. 2006). The 1.4 GHz GMRT image does not show any relevant structures and, therefore, the image is not shown.

The largest ring-shaped structure visible in the GMRT image of Fig. 1 follows the morphology of the SNR G35.6-0.4 (Green 2009). There is also a smaller ring-shaped structure at the southeast end of the SNR, appearing as an independent structure. This structure, not resolved in previous radio images, corresponds to an HII region that in the past led to consider G35.6-0.4 an H II region (Lockman 1989). Other relevant, compact, or slightly extended radio sources of this region detected above $5 \sigma$ are labelled with a number and are listed in Table 2. None of these five sources shows significant variability within uncertainties when comparing the flux density between June 4 and July 8 . The most relevant sources are described in more detail in what follows. 
J. M. Paredes et al.: Deep GMRT radio observations and a multi-wavelength study of the region around HESS J1858+020

Table 2. Radio sources detected in the HESS J1858+020 field with GMRT at $610 \mathrm{MHz}$ and $1.4 \mathrm{GHz}$.

\begin{tabular}{lccccrl}
\hline \hline Source & $\begin{array}{c}\alpha_{\mathrm{J} 2000.0} \\
{[\mathrm{~h} \mathrm{~m} \mathrm{~s}]}\end{array}$ & $\begin{array}{c}\delta_{\mathrm{J} 2000.0} \\
{\left[{ }^{\circ}{ }^{\prime \prime}\right]}\end{array}$ & $\begin{array}{c}S_{610 \mathrm{MHz}} \\
{[\mathrm{mJy}]}\end{array}$ & $\begin{array}{c}S_{1.4 \mathrm{GHz}} \\
{[\mathrm{mJy}]}\end{array}$ & $\begin{array}{c}\text { Spectral } \\
\text { index } \alpha\end{array}$ & Structure \\
\hline R1 & $185737.117 \pm 0.003$ & $021232.33 \pm 0.05$ & $24.3 \pm 1.1$ & $24.0 \pm 0.6$ & $-0.02 \pm 0.07$ & \\
R2 & $185737.955 \pm 0.002$ & $020340.49 \pm 0.03$ & $46.7 \pm 1.2$ & $118.5 \pm 2.0$ & $1.26 \pm 0.04$ & Planetary nebula \\
R3 & $185759.578 \pm 0.007$ & $020706.97 \pm 0.10$ & $50.6 \pm 1.0$ & $90.3 \pm 6.3$ & $0.78 \pm 0.10$ & Planetary nebula \\
R4 & $185812.557 \pm 0.003$ & $021652.94 \pm 0.04$ & $31.0 \pm 0.4$ & $24.8 \pm 0.5$ & $-0.30 \pm 0.03$ & \\
R5 & $185813.898 \pm 0.004$ & $021452.05 \pm 0.05$ & $21.7 \pm 0.2$ & $13.1 \pm 0.2$ & $-0.68 \pm 0.02$ & \\
\hline
\end{tabular}

Notes. We obtained the J2000.0 coordinates from the $1.4 \mathrm{GHz}$ map. The $610 \mathrm{MHz}$ flux density measurements were obtained from the combined image produced using data from June 4 and July 8. The spectral index $\alpha$ is defined as $S_{v} \propto v^{\alpha}$, where $S_{v}$ is the flux density at frequency $v$. The first column provides the assigned number to each source, ordered by increasing right ascension. The second and third columns list the J2000.0 right ascension and declination, respectively. The fourth and fifth columns provide the integrated flux density and its error at $610 \mathrm{MHz}$ and $1.4 \mathrm{GHz}$, respectively. The last column lists the structure of the sources.

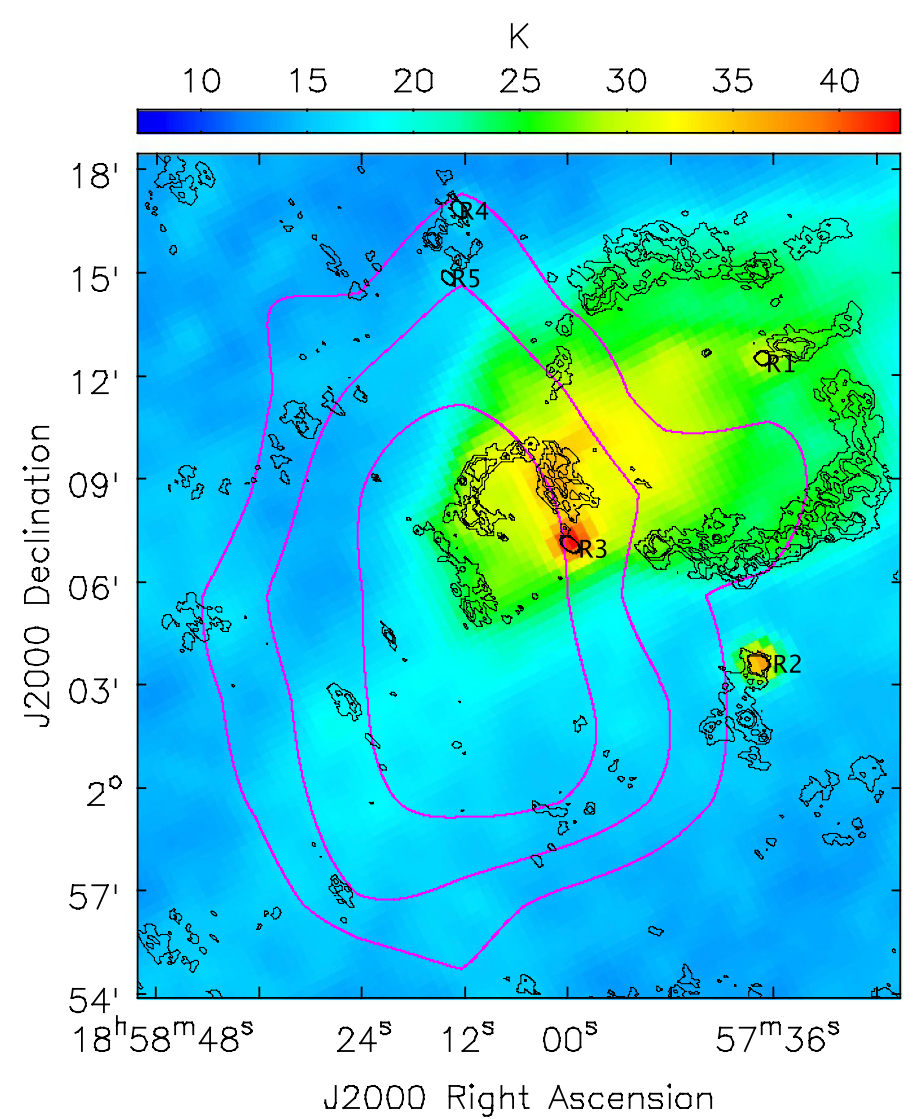

Fig. 1. GMRT $610 \mathrm{MHz}$ image (black contours). The contours correspond to 2,3 , and 4 times $0.22 \mathrm{mJy}$ beam $^{-1}$, the rms, and the beam size is $12.2^{\prime \prime} \times 4.8^{\prime \prime}$. The colour image shows the VGPS data of the SNR G35.6-0.4 at $1.4 \mathrm{GHz}$ with arcminute resolution (Stil et al. 2006). The HESS significance contours $(4,5$, and $6 \sigma$, in magenta) for the location of HESS J1858+020 are overlaid.

\subsection{Supernova remnant G35.6-0.4}

The extended radio source G35.6-0.4, previously thought to be an $\mathrm{H}$ II region, was recently identified as a SNR (Green 2009). Its morphology was revealed by the 1 arcmin resolution images from the VGPS at $1.4 \mathrm{GHz}$ (Stil et al. 2006) (see Fig. 1), and its non-thermal radio spectrum (spectral index $\alpha=-0.47 \pm 0.07$ ) was obtained using data from surveys with low resolution (7.8 Jy at $1.4 \mathrm{GHz}$ and of $3.1 \mathrm{Jy}$ at $10 \mathrm{GHz}$ ) (Green 2009). Observations of the H I line in the VGPS provided a lower limit to the distance of about $3.7 \mathrm{kpc}$ (Green 2009). A recent kinematic distance study of the complex where the SNR G35.6-0.4 is placed has established a distance to the SNR of $3.6 \pm 0.4 \mathrm{kpc}$ (Zhu et al. 2013).

Our image at $1.4 \mathrm{GHz}$ does not show the SNR structure because our high spatial resolution resolves it out. Our $610 \mathrm{MHz}$ image of the field containing HESS J1858+020 shown in Figs. 1 and 2 provides the highest resolution radio image to date (5-10 arcsec) of SNR G35.6-0.4, showing a nearly circular morphology $\left(10^{\prime} \times 8^{\prime}\right.$ or $\left.\approx 10 \times 8 \mathrm{pc}\right)$ that corresponds to the limb brightened region of emission. The integrated flux density of SNR G35.6-0.4 from our $610 \mathrm{MHz}$ image is $0.46 \mathrm{Jy}$.

Within the field of the SNR, there are two known pulsars that we have not detected. The pulsar PSR J1857+0212 (PSR B1855+02), with a period of $0.417 \mathrm{~s}$, was proposed to be associated with the remnant G35.6-0.4 (Phillips \& Onello 1993). The characteristic age is $\sim 1.6 \times 10^{5}$ yr (Hobbs et al. 2004) and the estimated distance, derived from the observed dispersion measure, is $\sim 8 \mathrm{kpc}$ (Hobbs et al. 2005). The other pulsar is PSR J1857+0210, which is close to the centre of the SNR, and has a flux density at $1.4 \mathrm{GHz}$ of $0.30(6) \mathrm{mJy}$, a period of $0.63 \mathrm{~s}$, a characteristic age of $7 \times 10^{5} \mathrm{yr}$, and an estimated distance of $15.4 \mathrm{kpc}$ (Morris et al. 2002). The expected age of the SNR, deduced from a Sedov-Taylor model, is about $3 \times 10^{4} \mathrm{yr}$, which poses difficulties for the association of the SNR with any of these pulsars (Green 2009). However, the not well established link between the characteristic and the true age of the pulsars prevents us from ruling out this association.

\subsection{The $H$ II region}

Radio recombination lines towards $l=35.588, b=-0^{\circ} .489$ were detected in a survey of radio H II regions in the northern sky (Lockman 1989). Further detections of recombination lines ( $\mathrm{H} 168 \alpha$ at $1374.6006 \mathrm{MHz}$ ) in the same region show broad lines indicative of enhanced turbulence caused by a non-thermal source (Phillips \& Onello 1993). This source could be the SNR G35.6-0.4 and this would imply that the HII is located at the same distance from the SNR, i.e., 3.6 $\pm 0.4 \mathrm{kpc}$ (Zhu et al. 2013). From our results shown in Fig. 2, we can see that the $\mathrm{H}$ II region is positionally coincident $\left(\alpha_{\mathrm{J} 2000.0}=18 \mathrm{~h} 58 \mathrm{~m}\right.$ and $\left.\delta_{\mathrm{J} 2000.0}=2^{\circ} 8^{\prime}\right)$ with the ring-shaped radio structure that appears in the southern corner of the SNR G35.6-0.4. The size of the ring is about 3', which corresponds to a linear size of 3 pc adopting the distance of $3.6 \mathrm{kpc}$. This structure seems to be morphologically independent of the SNR, although there could be some physical influence (turbulence) from the SNR. The H II region is nearly positionally coincident with the centroid of HESS J1858+020. 


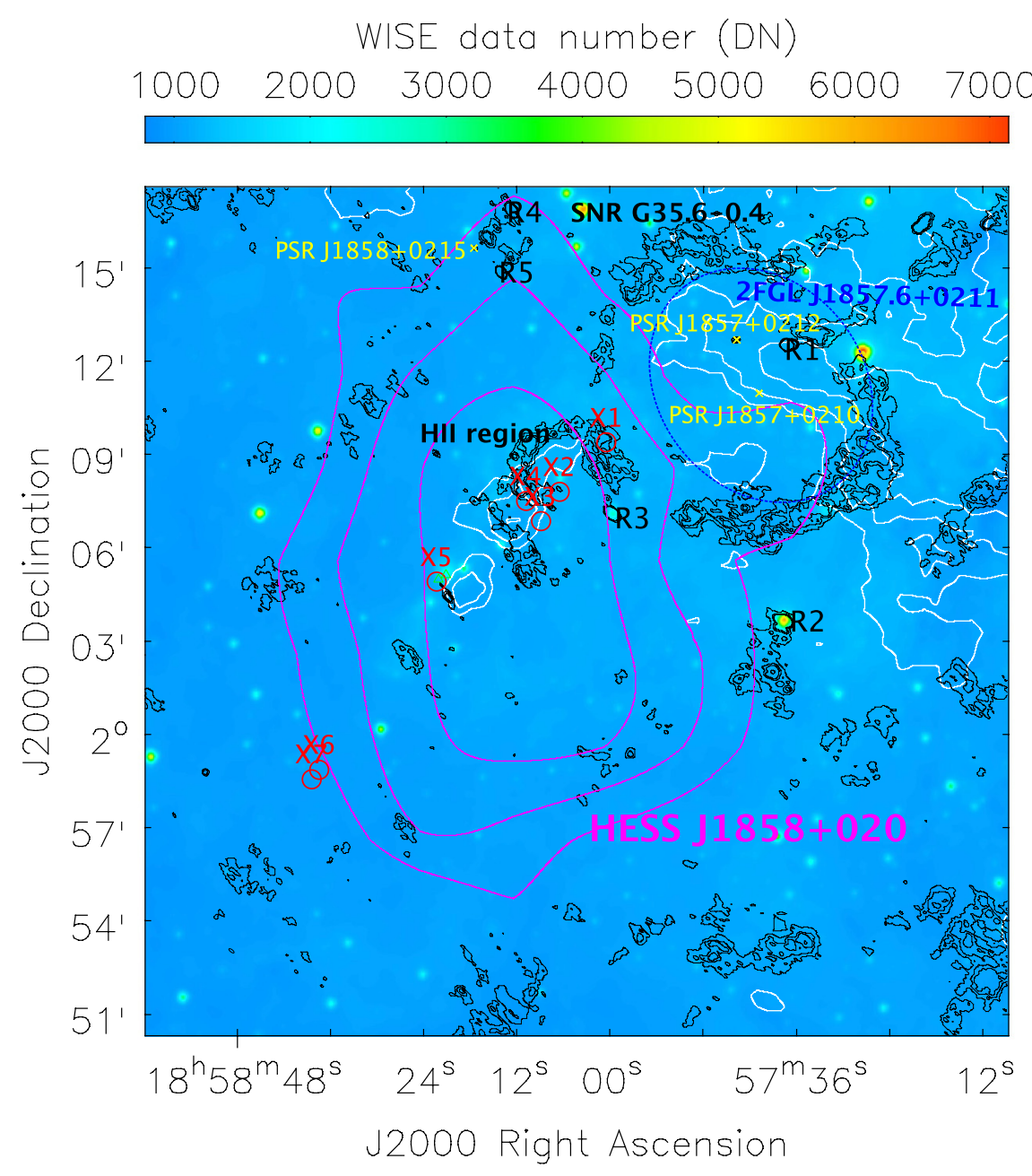

Fig. 2. Radio image (black contours) of the field containing the unidentified HESS source HESS J1858+020 obtained with GMRT at $610 \mathrm{MHz}$. The contours correspond to 2, 3, and 4 times $0.22 \mathrm{mJy}$ beam $^{-1}$, the rms, and the beam size is $12.2^{\prime \prime} \times 4.8^{\prime \prime}$. The colour image shows the $12 \mu \mathrm{m}$ WISE image of this region (Wright et al. 2010). The white contours show the ${ }^{13} \mathrm{CO}(J=1-0)$ emission integrated between 51 and $59 \mathrm{~km} \mathrm{~s}^{-1}$ from the Galactic Ring Survey (Jackson et al. 2006). The red circles correspond to X-ray sources. Some relevant radio sources are labelled as $\mathrm{R} 1, . ., \mathrm{R} 5$, respectively (see Table 2). The shell-like structure clearly visible in the upper right region of the figure corresponds to the SNR G35.6-0.4. The HESS significance contours $(4,5$, and $6 \sigma$, in magenta) for the location of HESS J1858+020 are overlaid. The blue ellipse indicates the 95\% confidence location of the Fermi-LAT gamma-ray source 2FGL J1857.6+0211. The positions of the three pulsars are indicated with yellow crosses, and the positions of two young stellar objects, close to X5, are indicated by triangles.

\subsection{Other sources}

Source R1: this source is clearly detected by GMRT and resolved at both frequencies, showing an elongated morphology of about 10 arcsec in length at $1.4 \mathrm{GHz}$ (see Fig. 3, top left) and a more symmetric morphology of about $25 \operatorname{arcsec}$ at $610 \mathrm{MHz}$. The flux density at $610 \mathrm{MHz}$ is $24.3 \pm 1.1 \mathrm{mJy}$ and at $1.4 \mathrm{GHz}$ is $25.2 \pm 0.7 \mathrm{mJy}$, implying a flat spectral index. This source also appears in the Small-Diameter Radio Sources Catalogue (Zoonematkermani et al. 1990) with an integrated flux density of $21 \mathrm{mJy}$ at $1.4 \mathrm{GHz}$, and in the Compact Radio Sources in the Galactic Plane Catalogue (White et al. 2005), with an integrated flux of $10.45 \mathrm{mJy}$ at $5 \mathrm{GHz}$. There is no counterpart in the 2MASS archive data. The source is outside the significance contours of HESS J1858+020, but within the Fermi-LAT source 2FGL J1857.6+0211 position error box (see Fig. 2).

Source R2: this is one of the strongest sources in the field. Its location, well outside the significance contours of HESS J1858+020 and the error box of 2FGL J1857.6+0211, makes this source an unlikely counterpart of these gamma-ray sources. Detected at both frequencies, $46.7 \pm 1.2 \mathrm{mJy}$ at $610 \mathrm{MHz}$ and $125 \pm 4 \mathrm{mJy}$ at $1.4 \mathrm{GHz}$, source R2 shows an elongated structure (see the top middle panel of Fig. 3). Our 1.4 GHz flux density is similar to the value of $117 \pm 4 \mathrm{mJy}$ obtained by Condon et al. (1999). Kistiakowsky \& Helfand (1995) catalogued the source 2MASS J18573787+0203440 (IRAS 18551+0159), positionally coincident with source R2, as a planetary nebula. Zhu et al. (2013) estimated a distance of $4.3 \pm 0.5 \mathrm{kpc}$ through kinematic distance studies.
Source R3: this radio source is positionally coincident with IRAS $18554+0203$. It has been detected clearly at both frequencies with the flux density at $1.4 \mathrm{GHz}$ nearly doubling the value obtained at $600 \mathrm{MHz}$. At both frequencies it has been resolved, showing a round structure of $\approx 12^{\prime \prime}$ at $1.4 \mathrm{GHz}$ (see the top right panel of Fig. 3) and $\approx 30^{\prime \prime}$ at $600 \mathrm{MHz}$. This source has been catalogued as a Galactic planetary nebula (PN G035.5-00.4, PHR 1857+0207), showing a radio flux density of $100 \pm 4 \mathrm{mJy}$ at $843 \mathrm{MHz}$ and an optically determined angular diameter in MASH of 11" (Bojičić et al. 2011). Zhu et al. (2013) estimated a lower limit distance of $3.8 \pm 0.4 \mathrm{kpc}$ and an upper limit distance of $5.4 \pm 0.7 \mathrm{kpc}$ through kinematic distance studies.

Source R4: this source has been resolved with GMRT at $1.4 \mathrm{GHz}$, showing a nearly north-south elongated structure (see the bottom left panel of Fig. 3). The source appears in the $20 \mathrm{~cm}$ source catalogue of compact radio sources in the Galactic plane (White et al. 2005) with an integrated flux density of $24 \mathrm{mJy}$ and with hints of northsouth elongation and with a flux density of $23 \pm 0.3 \mathrm{mJy}$ in the $5 \mathrm{GHz}$ CORNISH catalogue (Purcell et al. 2013). There are two optical/infrared sources, 2MASS 18581274+0216523 and 2MASS $18581239+0216513$, placed within the radio contours. Our measured spectral index (see Table 2) suggests a non-thermal nature.

Source R5: this source appears in the $20 \mathrm{~cm}$ source catalogue of compact radio sources in the Galactic plane with an integrated flux density of $6.7 \mathrm{mJy}$, whereas it appears resolved in 
J. M. Paredes et al.: Deep GMRT radio observations and a multi-wavelength study of the region around HESS J1858+020
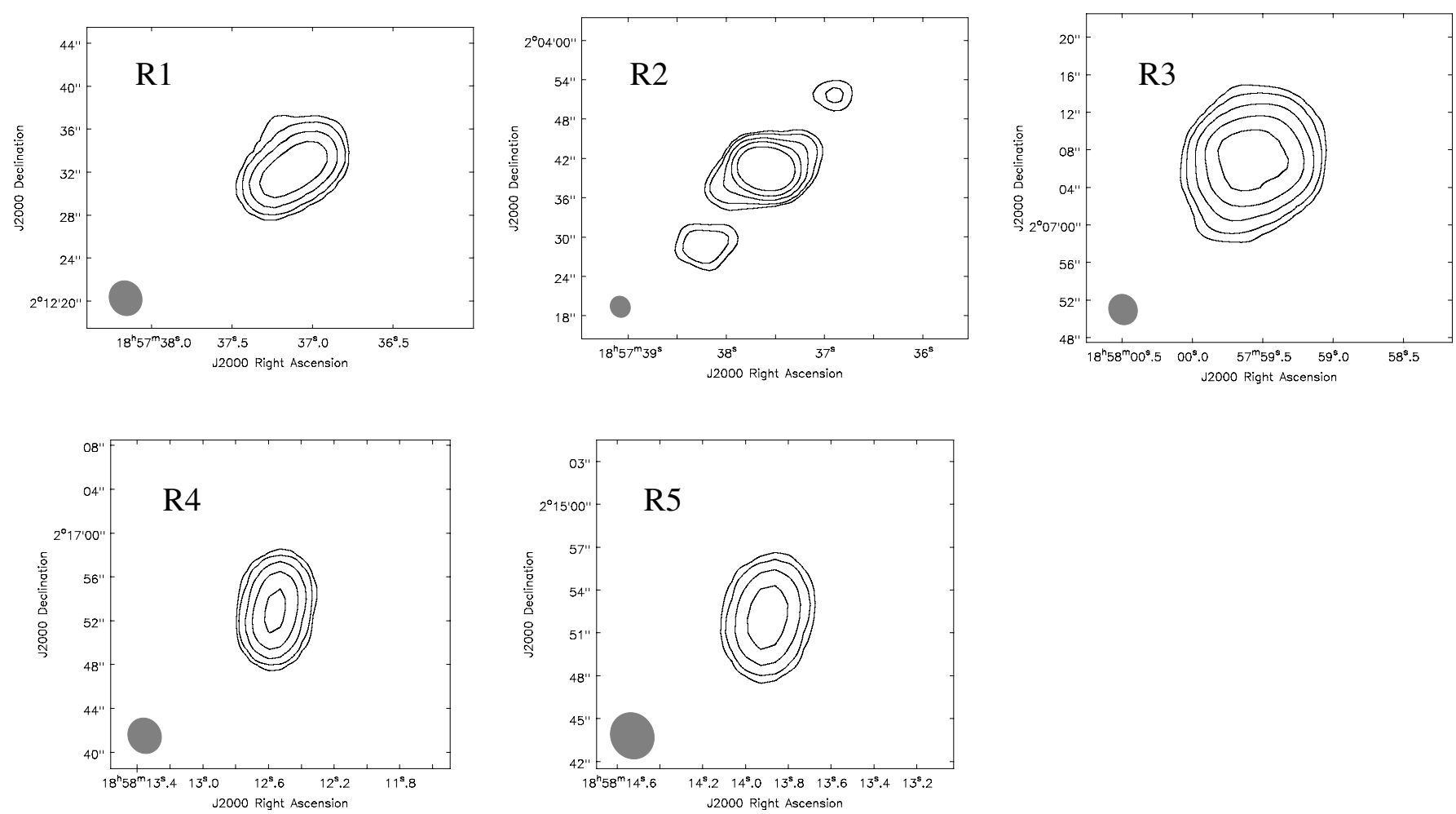

Fig. 3. GMRT image at $1.4 \mathrm{GHz}$ of the five sources listed in Table 2. The radio contours correspond to 5, 8, 16, 32, and 64 times $0.049 \mathrm{mJy}^{\text {beam }}{ }^{-1}$, the rms noise. The synthesized radio beam is plotted in the lower-left corner. Sources R2 and R3 are catalogued as possible and confirmed planetary nebula respectively.

our GMRT $1.4 \mathrm{GHz}$ observations (see the bottom middle panel of Fig. 3). In the CORNISH catalogue, this source has a flux density of $4.3 \pm 0.4 \mathrm{mJy}$ at $5 \mathrm{GHz}$. Our measured spectral index (see Table 2) suggests a non-thermal nature.

PSR J1858+0215: although we have not detected this object, we note the existence of this source, which is located in the northern part of HESS $\mathrm{J} 1858+020$, within its 4 and $5 \sigma$ confidence contour levels and very close to sources R4 and R5. Its flux density at $1.4 \mathrm{GHz}$ is $0.22(4) \mathrm{mJy}$, the period is $0.75 \mathrm{~s}$, the age is of about $2.6 \times 10^{6}$ years, and the distance is $12.4 \mathrm{kpc}$ (Morris et al. 2002).

\section{Multi-wavelength study}

The results obtained from the analysis of archival data and the data from different surveys, as well as our GMRT radio data, are shown in Fig. 2.

\subsection{Molecular cloud}

Paron \& Giacani (2010) and Paron et al. (2011) revealed the existence of a molecular cloud, formed by two clumps, in the central region of HESS J1858+020, using the ${ }^{13} \mathrm{CO}(J=1-0)$ line from the Galactic Ring Survey. The northern clump shows kinematical hints of shocked gas that could evidence turbulent motion of the gas. This turbulent motion was suggested to be caused by the interaction of the SNR G35.6-0.4 with the molecular cloud (Paron \& Giacani 2010). In Fig. 2, we can see that the ring-shaped structure detected in our $610 \mathrm{MHz}$ observations overlaps with the northern clump, suggesting an association between the $\mathrm{H}$ II region and the molecular cloud. In fact, the study of the impact of stellar winds, supernovae, and $\mathrm{H}$ II regions onto cloud turbulence shows that $\mathrm{H}$ II regions most efficiently sustain this turbulence (Matzner 2002). On other hand, kinematic absorption features shown in Zhu et al. (2013) make a common distance for both the molecular cloud and the supernova plausible.

The southern molecular clump has no radio counterpart, as can be seen in Fig. 2. However, Paron et al. (2011) identified two intrinsically red sources in the infrared domain, named IRS 1 $\left(\alpha_{\mathrm{J} 2000.0}=18 \mathrm{~h} 58 \mathrm{~m} 21.15 \mathrm{~s}\right.$ and $\left.\delta_{\mathrm{J} 2000.0}=2^{\circ} 5^{\prime} 10.0^{\prime \prime}\right)$ and IRS2 $\left(\alpha_{\mathrm{J} 2000.0}=18 \mathrm{~h} 58 \mathrm{~m} 22.12 \mathrm{~s}\right.$ and $\left.\delta_{\mathrm{J} 2000.0}=2^{\circ} 5^{\prime} 1.4^{\prime \prime}\right)$, which appear to be related to the molecular cloud. These authors suggest that IRS1 is an evolved young stellar object, probably in the last stages of formation, which could be a high-mass protostellar object embedded in the southern molecular clump. It appears as a bright point source (mag $6.51 \pm 0.03$ ) in the $12 \mu \mathrm{m}$ image.

\subsection{Chandra $X$-ray sources}

In the 0.4-7 keV image taken with Chandra around the HESS source, seven X-ray sources are detected by celldetect at significance above the conservative $3 \sigma$ threshold. The locations of these sources are indicated in Fig. 2 by red circles. Table 3 lists the position, $0.4-7 \mathrm{keV}$ net counts, the significance of the celldetect detection, and the hardness ratio defined as $H R=(H-S) /(H+S)$ (where $H$ and $S$ are source counts in the 3-7 keV and 1-3 keV bands, respectively). The HR of X1, 2-4 all have positive values, implying significantly harder spectra than X5 and X6. None of the seven sources has an IR counterpart. Four of the detected sources, X1, 2-4, coincide with the northern molecular clump and the ring-shaped radio structure.

As their HR suggest, X1-4 have similar spectra and the combined (a straight sum) spectrum of the four sources (Fig. 4) shows strong absorption and a hint of an $\mathrm{Fe} \mathrm{K}$ emission line 
Table 3. X-ray sources detected after the analysis of archival Chandra data covering the central region of HESS J1858+020.

\begin{tabular}{lccccr}
\hline \hline Source & $\begin{array}{c}\alpha_{\mathrm{J} 2000.0} \\
{[\mathrm{~h} \mathrm{~m} \mathrm{~s}]}\end{array}$ & $\begin{array}{c}\delta_{\mathrm{J} 2000.0} \\
{\left[{ }^{\circ}{ }^{\prime}{ }^{\prime}\right]}\end{array}$ & $\begin{array}{c}\text { Counts } \\
{[0.4-7 \mathrm{keV}]}\end{array}$ & Significance & \multicolumn{1}{l}{$H R$} \\
\hline $\mathrm{X} 1$ & 185800.46 & 020923.3 & $35.04 \pm 8.27$ & 4.23 & $0.38 \pm 0.18$ \\
$\mathrm{X} 2$ & 185806.49 & 020747.7 & $22.43 \pm 6.97$ & 3.22 & $0.72 \pm 0.24$ \\
$\mathrm{X} 3$ & 185808.97 & 020651.2 & $22.24 \pm 6.65$ & 3.34 & $0.55 \pm 0.23$ \\
$\mathrm{X} 4$ & 185810.71 & 020730.1 & $40.14 \pm 8.57$ & 4.69 & $0.45 \pm 0.16$ \\
$\mathrm{X} 5$ & 185822.21 & 020454.6 & $64.43 \pm 9.94$ & 6.55 & $-0.13 \pm 0.12$ \\
$\mathrm{X} 6$ & 185837.38 & 015851.5 & $62.24 \pm 9.50$ & 6.55 & $-0.80 \pm 0.20$ \\
$\mathrm{X} 7$ & 185838.50 & 015833.2 & $20.69 \pm 6.80$ & 3.04 & $0.05 \pm 0.22$ \\
\hline
\end{tabular}

at $6.53 \pm 0.09 \mathrm{keV}($ at $\sim 1.5 \sigma)$. If this Fe $\mathrm{K}$ line were real, it would suggest a thermal origin of the X-ray emission. Whereas the temperature and absorption are degenerate in spectral fit and are not well constrained, when solar abundances are assumed, the likely temperature of the thermal emitting gas is $k T \sim 1.5-2 \mathrm{keV}$ (which provides a good description of the Fe K line energy and strength) with an absorbing column density of $N_{\mathrm{H}} \sim(1-1.5) \times 10^{23} \mathrm{~cm}^{-2}$. These spectral properties suggest that these sources might be embedded protostars. The mean flux of these sources as observed in the $2-7 \mathrm{keV}$ band is $2.6 \times 10^{-14} \mathrm{erg} \mathrm{s}^{-1} \mathrm{~cm}^{-2}$.

The brightest source in the $0.4-7 \mathrm{keV}$ band, $\mathrm{X} 5$, is weaker than X1-4 in the 3-7 keV band. A power-law of in$\operatorname{dex} \Gamma=2.1 \pm 0.6$ and a thermal spectrum with $k T=$ $3.2 \pm 1.1 \mathrm{keV}$ yield comparable fits to the data (Fig. 5). The absorption $N_{\mathrm{H}}=(1.5 \pm 0.6) \times 10^{22} \mathrm{~cm}^{-2}$ agrees with the typical Galactic absorption towards this direction of the sky for a distance of several kpc. The observed 2-7 keV flux is $2.7 \times 10^{-14} \mathrm{erg} \mathrm{s}^{-1} \mathrm{~cm}^{-2}$. This source is potentially coincident with the star forming region and close to the southern molecular clump.

The source X6 has the softest spectrum and, unlike other sources, significant emission is detected below $1 \mathrm{keV}$ indicating low absorption. This probably means that this is a foreground source. The faintest of the X-ray sources detected is X7, and it is not possible to get a reliable spectrum.

Besides the point-like sources, diffuse emission, possibly associated with HESS J1858+020, was searched in the Chandra data. As such diffuse emission could have an extension on arcmin-scale, the X-ray image coarsely binned with various angular scales was inspected, but no clear extended emission was found. Based on the statistical fluctuation of the background of the field, the $2 \sigma$ upper limit was obtained to be $\sim 1 \times$ $10^{-13} \mathrm{erg} \mathrm{cm}^{-2} \mathrm{~s}^{-1}$ for a source with a $6^{\prime} \times 6^{\prime}$ extension and an X-ray spectrum of a power-law of photon-index $\Gamma=2$ (the flux was corrected for the Galactic absorption, approximated to be $N_{\mathrm{H}}=1.5 \times 10^{22} \mathrm{~cm}^{-2}$ ).

\subsection{The high energy gamma-ray source 2FGL J1857.6+0211}

The source 2FGL J1857.6+0211 is positionally the closest Fermi-LAT source to HESS J1858+020 that appears in the Fermi Large Area Telescope Second Source Catalogue (Nolan et al. 2012). However, a likelihood analysis performed to investigate a possible association between HESS J1858+020 and 2FGL J1857.6+0211 (Torres et al. 2011) suggests that this association is unlikely (see however Sect. 6). The Fermi-LAT source, with a semi-major and semi-minor axis of position error ellipse at $95 \%$ confidence of $4^{\prime} \times 3^{\prime}$, remains unidentified.

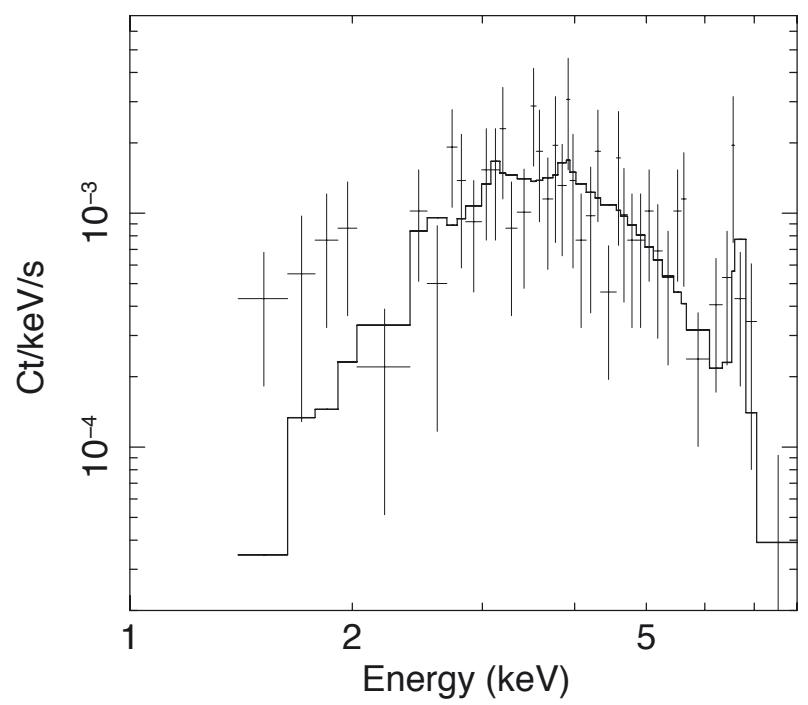

Fig. 4. X-ray spectrum of the summed X1-4 sources, obtained from Chandra ACIS-I. No X-ray emission below $1 \mathrm{keV}$ is detected. The solid-line histogram shows the folded model of a thermal emission spectrum of $k T=2 \mathrm{keV}$ modified by absorption of $N_{\mathrm{H}}=$ $1.1 \times 10^{23} \mathrm{~cm}^{-2}$.

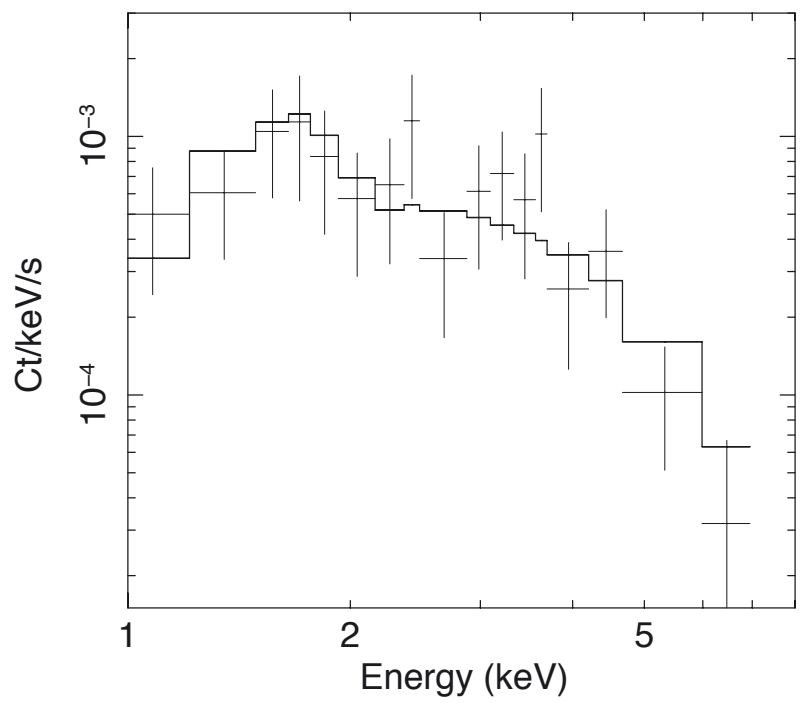

Fig. 5. X-ray spectrum of X5. No significant X-ray emission is detected below $1 \mathrm{keV}$. The solid-line histogram indicates the folded model of a power-law of $\Gamma=2.1$ modified by absorption with $N_{\mathrm{H}}=$ $1.5 \times 10^{22} \mathrm{~cm}^{-2}$.

The pulsars PSR J1857+0212 and PSR J1857+0210 are both located well inside the 2FGL J1857.6+0211 error box. Fermi-LAT has not yet detected pulsed gamma-ray emission from any of the pulsars.

\section{Discussion}

It has been proposed that the $\mathrm{TeV} \gamma$-ray emission detected by HESS has a hadronic origin and is produced by the interaction between cosmic rays accelerated by the shock front of the SNR and a nearby molecular cloud (Paron et al. 2011). Torres et al. (2011) explored this possibility through the study of the parameter space considering different cloud masses, cloud-SNR distances, and spatial diffusion coefficients. They concluded that only a rather small diffusion coefficient, $\sim 100$ times lower 
than the Galactic one, and a short distance from the SNR, of about $40 \mathrm{pc}$, could reconcile the detection of TeV emission, the $\mathrm{GeV}$ upper limits at the location of the HESS source, and the cloud mass derived by Paron \& Giacani (2010). Nevertheless, given the small projected distance of $\sim 5 \mathrm{pc}$ (at a distance of $3.6 \mathrm{kpc}$ ) between the cloud and the SNR, plus the fact that close to SNR shocks particle propagation may be significantly slowed down (Fujita et al. 2011; Malkov et al. 2013), the connection between the TeV emission and the SNR cannot be discarded. In fact, new calculations modelling the interaction between the SNR cosmic rays and the cloud, using the distance of $3.6 \mathrm{kpc}$, relax the conditions on the diffusion coefficient and cloud mass, making this possibility more likely than previously thought (see discussion in Zhu et al. 2013). The X-ray upper limit for the SNR G35.6-0.4 and MC interaction region we found can help us to constrain, in future modelling, the physical conditions of the TeV emitter, like the magnetic field and the medium density.

The age of the SNR, probably close to its transition to the radiative phase, but possibly still under adiabatic expansion (Torres et al. 2011), suggests that it could still accelerate protons energetic enough to produce $\mathrm{GeV}$ gamma rays. The recently estimated distance of $3.6 \mathrm{kpc}$ makes the energetics of the source less tight and favors this possibility. The SNR origin could explain the co-spatial Fermi-LAT source 2FGL J1857.6+0211, as in other middle-aged SNR, through interactions with very nearby clouds or shock-embedded dense material (see, e.g., Ajello et al. 2012, and references therein). Therefore, it seems feasible that SNR G35.6-0.4 would be the origin of both the GeV and $\mathrm{TeV}$ emission.

Other sources presented in this work may be behind HESS J1858+020 and/or 2FGL J1857.6+0211, although their association seems to be less plausible than SNR G35.6-0.4. There are the three pulsars discussed above. Being rather old and far, with distances $\gtrsim 10 \mathrm{kpc}$, their spin-down luminosities are certainly not enough to power the $\mathrm{GeV}$ emission centred at the location of the SNR, and may be insufficient to explain the $\mathrm{TeV}$ emission. In addition, all three pulsars lie outside the significance contours of the HESS source, making their connection with the $\mathrm{TeV}$ radiation even more unlikely. However, one of these objects may still be behind an ancient pulsar wind nebula, with the pulsar being off-set and having a complex morphology and no X-ray radiation (see, e.g., Kargaltsev \& Pavlov 2010), although the age may argue against an association with 2FGL J1857.6+0211 (Kargaltsev et al. 2013). The two planetary nebulae also present in the region can be discarded as counterparts of the gamma-ray sources as well. These objects, remnants of low-to-intermediate mass stars, would have limited energetics and relatively low speeds, which are unsuitable conditions to accelerate particles up to very high energies. The two non-thermal radio sources ( $\mathrm{R} 4$ and $\mathrm{R} 5$ ), with unclear counterpart at other wavelengths, might be candidates for high energy emitters, although at present assessing this statement requires further study to identify their physical origin. Finally, the star forming region deserves some attention, in particular the elongated ${ }^{13} \mathrm{CO}$ structure and several $\mathrm{X}$-ray sources with positions roughly aligned with the former. As mentioned, this region could host a number of protostars, with at least one among them possibly of high mass nature. Moreover, the apparent elongation of the region may be hinting at a powerful outflow, which could be masked in radio by strong free-free absorption. Such an outflow, and the entire star formation region, could be the origin of, or contribute to, HESS J1858+020 (e.g. Bosch-Ramon et al. 2010). However, this possibility requires further observational studies to sustain the case for strong interactions in the star formation region as well.

\section{Conclusions}

The region surrounding HESS J1858+020 is complex. For instance, there are several molecular structures, possibly linked with different types of non-thermal objects, like SNR or star forming regions, and these are suitable environments for the formation of high energy emission. In this regard, several candidates can be identified as potential counterparts to the HESS (and possibly also the Fermi) source: a SNR interacting with very near molecular clouds through a medium with a low diffusion coefficient; a star forming region with several young stellar objects, some of them possibly of high mass; and non-thermal extended radio sources that may be Galactic in origin and host relativistic jets. Finally, an ancient pulsar wind nebula, despite the far distance of the pulsars neighbouring the HESS source, cannot be discarded as a candidate. In any case, given the natural uncertainties when determining the most likely candidate associated with the richness of the presented results, further observational studies are needed to narrow the search for a counterpart to HESS J1858+020.

Acknowledgements. J.M.P., V.B.-R., V.Z. and M.R. acknowledge support by DGI of the Spanish Ministerio de Economía y Competitividad (MINECO) under grants AYA2010-21782-C03-01 and FPA2010-22056-C06-02. V.B.-R. acknowledges financial support from MINECO through a Ramón y Cajal fellowship. J.M.P. acknowledge financial support from ICREA Academia. V.Z. acknowledges support by the Generalitat de Catalunya through the Beatriu de Pinós program, and by the Max-Planck-Gesellschaft. This research has been supported by the Marie Curie Career Integration Grant 321520. We thank the staff of GMRT who have made these observations possible. GMRT is run by the National Centre for Radio Astrophysics of the Tata Institute of Fundamental Research. This publication makes use of data products from the Wide-field Infrared Survey Explorer, which is a joint project of the University of California, Los Angeles, and the Jet Propulsion Laboratory/California Institute of Technology, funded by the National Aeronautics and Space Administration. This publication makes use of molecular line data from the Boston University-FCRAO Galactic Ring Survey (GRS). The GRS is a joint project of Boston University and Five College Radio Astronomy Observatory, funded by the National Science Foundation under grants AST-9800334, AST-0098562, AST-0100793, AST-0228993, and AST-0507657.

\section{References}

Aharonian, F., Akhperjanian, A. G., Bazer-Bachi, A. R., et al. 2006, ApJ, 636, 777

Aharonian, F., Akhperjanian, A. G., Bazer-Bachi, A. R., et al. 2007, A\&A, 467, 1075

Aharonian, F., Akhperjanian, A. G., Barres de Almeida, U., et al. 2008, A\&A, 477,353

Ajello, M., Allafort, A., Baldini, L., et al. 2012, ApJ, 744, 80

Araudo, A. T., Romero, G. E., Bosch-Ramon, V., \& Paredes, J. M. 2007, A\&A, 476, 1289

Bosch-Ramon, V., Romero, G. E., Araudo, A. T., \& Paredes, J. M. 2010, A\&A, 511, 8

Bojičić, I. S., Parker, Q. A., Filipović, M. D., \& Frew, D. J. 2011, MNRAS, 412, 223

Condon, J. J., Cotton, W. D., Greisen, E. W., et al. 1998, AJ, 115, 1693

Condon, J. J., Kaplan, D. L., \& Terzian, Y. 1999, ApJS, 123, 219

Fujita, Y., Takahara, F., Ohira, Y., \& Iwasaki, K. 2011, MNRAS, 415, 3434

Green, D. A. 2009, MNRAS, 399, 177

Green, A. J., Cram, L. E., Large, M. I., \& Ye, T. 1999, ApJS, 122, 207

Hobbs, G., Lyne, A. G., Kramer, M., Martin, C. E., \& Jordan, C. 2004, MNRAS, 353,1311

Hobbs, G., Lorimer, D. R., Lyne, A. G., \& Kramer, M. 2005, MNRAS, 360, 974

Jackson, J. M., Rathborne, J. M., Shah, R. Y., et al. 2006, ApJS, 163, 145 
Kargaltsev, O., \& Pavlov, G. G. 2010, AIP Conf. Proc., 1248, 25

Kargaltsev, O., Rangelov, B., \& Pavlov, G. G. 2013 [arXiv: 1305. 2552]

Kistiakowsky, V., \& Helfand, D. J. 1995, AJ, 110, 2225

Lockman, F. J. 1989, ApJS, 71, 469

Malkov, M. A., Diamond, P. H., Sagdeev, R. Z., Aharonian, F. A., \&

Moskalenko, I. V. 2013, ApJ, 768, 73

Matzner, C. D. 2002, ApJ, 566, 302

Morris, D. J., Hobbs, G., Lyne, A. G., et al. 2002, MNRAS, 335, 275

Murphy, T., Mauch, T., Green, A., et al. 2007, MNRAS, 382, 382

Nolan, P. L., Abdo, A. A., Ackermann, M., et al. 2012, ApJS, 199, 31

Paron, S., \& Giacani, G. 2010, A\&A, 509, L4

Paron, S., Giacani, E., Rubio, M., \& Dubner, G. 2011, A\&A, 530, A25

Phillips, J. A., \& Onello, J. S. 1993, in Massive Stars: Their Lives in the Interstellar Medium, ASP Conf. Ser., 35, 419

Pletsch, H. J., Guillemot, L., Allen, B., et al. 2012, ApJ, 755, L20
Purcell, C. R., Hoare, M. G., Cotton, W. D., et al. 2013, ApJS, 205, 1

Stamatescu, V., Krause, J., Klepser, S., et al. 2012, AIP Conf. Ser., 1505, 345

Stil, J. M., Taylor, A. R., Dickey, J. M., et al. 2006, AJ, 132, 1158

Tian, W. W., Leahy, D. A., Haverkorn, M., \& Jiang, B. 2008, ApJ, 679, L85

Tian, W. W., Li, Z., Leahy, D. A., et al. 2010, ApJ, 712, 790

Tibolla, O., Vorster, M., de Jager, O., et al. 2012, AIP Conf. Ser., 1505, 349

Torres, D. F., Romero, G. E., Dame, T. M., Combi, J. A., \& Butt, Y. M. 2003, Phys. Rep., 382, 303

Torres, D. F., Li, H., Chen, Y., et al. 2011, MNRAS, 417, 3072

Van Etten, A., Funk, S., \& Hinton, J. 2009, ApJ, 707, 1717

White, R. L., Becker, R. H., \& Helfand, D. J. 2005, AJ, 130, 586

Wright, E. L., Eisenhardt, P. R. M., Mainzer, A. K., et al. 2010, AJ, 140, 1868

Zhu, H., Tian, W. W., Torres, D. F., Pedaletti, G., \& Su, H. Q. 2013, ApJ, 775, 95

Zoonematkermani, S., Helfand, D. J., Becker, R. H., White, R. L., \& Perley, R. A. 1990, ApJS, 74, 181 